Supporting information for:

\title{
Structural Motifs of Wheat Straw Lignin Differ in Susceptibility to Degradation by the White-Rot Fungus Ceriporiopsis subvermispora
}

Gijs van Erven ${ }^{\mathrm{a}}$, Jianli Wang ${ }^{\mathrm{a}}$, Peicheng Sun ${ }^{\mathrm{a}}$, Pieter de Waard ${ }^{\mathrm{b}}$, Jacinta van der Putten ${ }^{\mathrm{c}}$, Guus E. Frissen ${ }^{\mathrm{c}}$, Richard J. A. Gosselink ${ }^{\mathrm{c}}$, Grigory Zinovyev ${ }^{\mathrm{d}}$, Antje Potthast ${ }^{\mathrm{d}}$, Willem J. H. van Berkel $^{\mathrm{a}}$, Mirjam A. Kabel ${ }^{\mathrm{a}^{*}}$

${ }^{a}$ Wageningen University \& Research, Laboratory of Food Chemistry, Bornse Weilanden 9, 6708 WG, Wageningen, The Netherlands.

${ }^{\mathrm{b}}$ MAGNEFY (MAGNEtic Resonance Research FacilitY), Wageningen University \& Research, Stippeneng 4, 6708 WE, Wageningen, The Netherlands

${ }^{\mathrm{c}}$ Wageningen Food and Biobased Research, Bornse Weilanden 9, 6708 WG, Wageningen, The Netherlands

${ }^{\mathrm{d} U n i v e r s i t y ~ o f ~ N a t u r a l ~ R e s o u r c e s ~ a n d ~ L i f e ~ S c i e n c e s, ~ D e p a r t m e n t ~ o f ~ C h e m i s t r y, ~ D i v i s i o n ~ o f ~}$ Chemistry of Renewable Resources, Konrad-Lorenz-Strasse 24, A-3430, Tulln, Austria

*Corresponding author. Tel: +31 317483209 + E-mail address: mirjam.kabel@wur.nl

Number of pages: 14

Number of figures: 2

Number of tables: 8 


\section{Supporting Information contents:}

\section{Experimental details carbohydrate content and composition}

Figure S1. Lignin, glucuronoarabinoxylan (GAX) and glucan (cellulose) removal during fungal growth.

Figure S2. SEC analysis of lignin isolates.

Table S1. ${ }^{13} \mathrm{C}-\mathrm{IS}$ py-GC-MS relative abundance of unfractionated wheat straw.

Table S2. Composition of lignin isolates.

Table S3. ${ }^{13} \mathrm{C}$-IS py-GC-MS relative abundance of ball-milled water extracted residue R4.

Table S4. ${ }^{13} \mathrm{C}-\mathrm{IS}$ py-GC-MS relative abundance of lignin isolates.

Table S5. ${ }^{13} \mathrm{C}-\mathrm{IS}$ py-GC-MS relative abundance of unextractable residue R7.

Table S6. Assignments of correlation peaks in HSQC spectra of lignin isolates.

Table S7. Semi-quantitative HSQC NMR analysis of lignin isolates in presence and absence of $\mathrm{Cr}(\mathrm{acac})_{3}$.

Table S8. Hydroxyl group content of lignin isolates of wheat straw during growth of $C$. subvermispora determined by ${ }^{31} \mathrm{P}$ NMR after phosphitylation.

\section{References}


Carbohydrate content and composition. Carbohydrate content and composition was determined in duplicate as constituent monosaccharides after acid hydrolysis by a modified method reported by Englyst \& Cummings. ${ }^{1}$ Ten $\mathrm{mg}$ of each sample was treated with $72 \%$ (w/w) $\mathrm{H}_{2} \mathrm{SO}_{4}$ for $1 \mathrm{~h}$ at $30{ }^{\circ} \mathrm{C}$ followed by $1 \mathrm{M} \mathrm{H}_{2} \mathrm{SO}_{4}$ for $3 \mathrm{~h}$ at $100{ }^{\circ} \mathrm{C}$. Subsequently, samples were centrifuged $\left(9,000 \mathrm{xg}, 5 \mathrm{~min}, 20^{\circ} \mathrm{C}\right)$ and the supernatants were diluted $(20 \mathrm{x})$ before analysis. Degradation of monosaccharides during hydrolysis was corrected for by including monosaccharide standard mixtures in hydrolysis. Analysis was performed on a High Performance Anion Exchange Chromatography (HPAEC) Dionex ICS-5000 system (Thermo Scientific, Synnyvale, CA, USA). The system was equipped with a CarboPac PA-1 column (250 mm x $2 \mathrm{~mm} \mathrm{ID)} \mathrm{in} \mathrm{combination} \mathrm{with} \mathrm{a} \mathrm{CarboPac} \mathrm{guard} \mathrm{column} \mathrm{(50} \mathrm{mm} \mathrm{x} 2 \mathrm{~mm}$ ID) with pulsed amperometric detection (PAD) (all Dionex). $10 \mu \mathrm{L}$ of sample was injected and eluted at a flow rate of $0.4 \mathrm{~mL} \cdot \mathrm{min}^{-1}$ using a combination of three mobile phases: A) $0.1 \mathrm{M}$ $\mathrm{NaOH}$, B) $1 \mathrm{M} \mathrm{NaOAc}$ in $0.1 \mathrm{M} \mathrm{NaOH}$ and C) $\mathrm{H}_{2} \mathrm{O}$. The elution profile used was as follows: 0-35 min isocratic on $100 \% \mathrm{C}$; $35-50$ min linearly from $100 \%$ A to $40 \% \mathrm{~B}$; $50-55 \mathrm{~min}$ isocratic on $100 \% \mathrm{~B}$; $55-63$ min isocratic on $100 \% \mathrm{~A}$; $63-78$ min isocratic on $100 \% \mathrm{C}$. Postcolumn addition of $0.5 \mathrm{M} \mathrm{NaOH}$ at $0.1 \mathrm{~mL} \cdot \mathrm{min}^{-1}$ was performed between $0-35 \mathrm{~min}$ and $63-78$ min. Data was processed by using Chromeleon 7 (Thermo Scientific). The uronic acids released after the acid hydrolysis step, were determined in duplicate as anhydrouronic acid content by an automated meta-hydroxydiphenyl assay with addition of sodium tetraborate using an auto-analyzer (Skalar Analytical BV, Breda, The Netherlands). ${ }^{2}$ Glucuronic acid (Fluka AG, Busch, Switzerland) was used as a reference $\left(0-100 \mu \mathrm{g} \mathrm{mL} \mathrm{m}^{-1}\right)$. Total carbohydrate content was calculated as the sum of neutral anhydrocarbohydrates and anhydrouronic acids. 


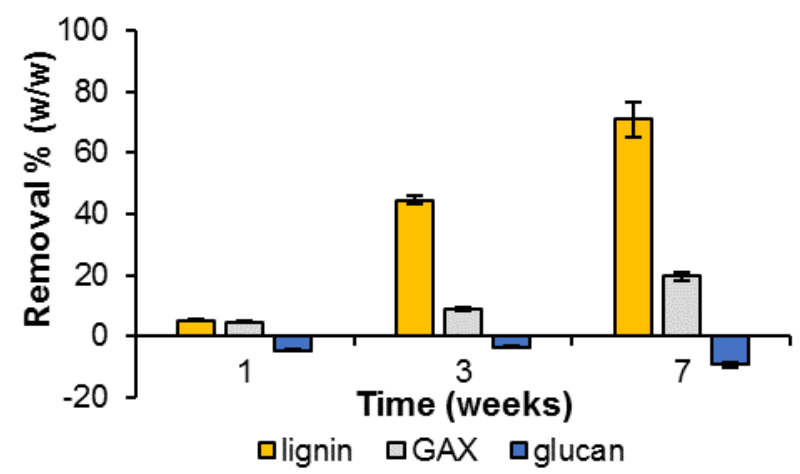

Figure S1. Lignin, glucuronoarabinoxylan (GAX) and glucan (cellulose) removal during fungal growth. Compositional analysis by using quantitative ${ }^{13} \mathrm{C}-\mathrm{IS}$ py-GC-MS (lignin) and constituent monosaccharide analysis after $\mathrm{H}_{2} \mathrm{SO}_{4}$ hydrolysis (carbohydrates). Average and standard deviation of analytical triplicates on pooled biological triplicates. The negative values for glucan are caused by the production of fungal $\beta$-glucan, which cannot be distinguished from cellulose in constituent monosaccharide analysis. Note that the 7 weeks fungal treated sample has been previously analyzed and reported in van Erven et al. (2018). ${ }^{3}$ This time-point was reanalyzed for this work.

Compositional analyses showed that lignin degradation by $C$. subvermispora was evident from the first week of growth on wheat straw (Figure S1). In the early stage, lignin and hemicellulose (glucuronoarabinoxylan, GAX) were removed to similar extent $(\sim 5 \%(\mathrm{w} / \mathrm{w}))$, while cellulose appeared unaffected. The relatively unselective action in the initial stage of growth is explained by the availability of (more) easily degradable carbohydrates. As soon as these were depleted, lignin degradation became more selective. After 3 weeks of growth, up to $45 \%(w / w)$ lignin was removed at the expense of $9 \%(w / w)$ GAX. During further growth, delignification continued and reached $71 \%$ (w/w) after 7 weeks, while GAX removal increased to $19 \%(\mathrm{w} / \mathrm{w}){ }^{3}$ Still, after 7 weeks of treatment more than $90 \%$ of the initial carbohydrates were retained in the residue, from which it is concluded that $C$. subvermispora delignified wheat straw in a highly effective and selective manner. 

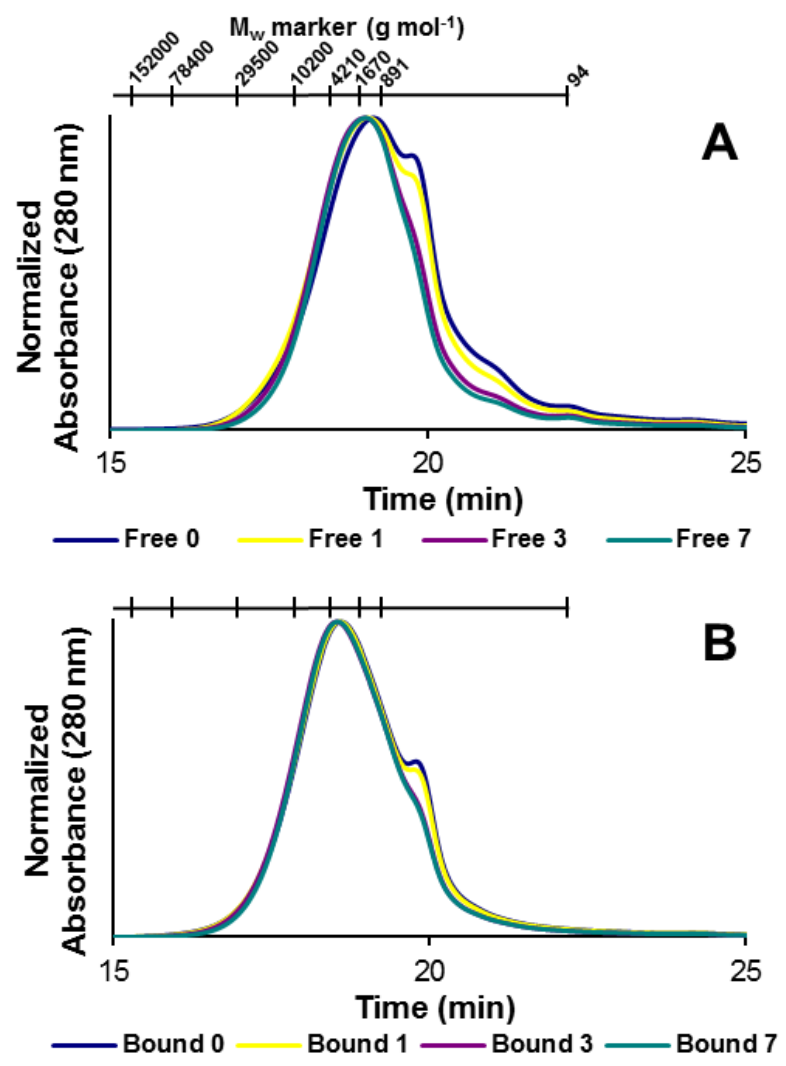

\begin{tabular}{cccc}
\hline & $\mathbf{M}_{\mathbf{n}}\left(\mathbf{g ~ m o l}^{-1}\right)$ & $\mathbf{M}_{\mathrm{w}}\left(\mathrm{g} \mathrm{mol}^{-1}\right)$ & $\mathbf{M}_{\mathrm{w}} / \mathbf{M}_{\mathbf{n}}$ \\
\hline Free 0 & 1015 & 6855 & 6.8 \\
Free 1 & 1120 & 7370 & 6.6 \\
Free 3 & 1040 & 6355 & 6.1 \\
Free 7 & 965 & 5355 & 5.5 \\
Bound 0 & 2265 & 13310 & 5.9 \\
Bound 1 & 2550 & 13480 & 5.3 \\
Bound 3 & 2520 & 14190 & 5.6 \\
Bound 7 & 2400 & 11245 & 4.7 \\
\hline
\end{tabular}

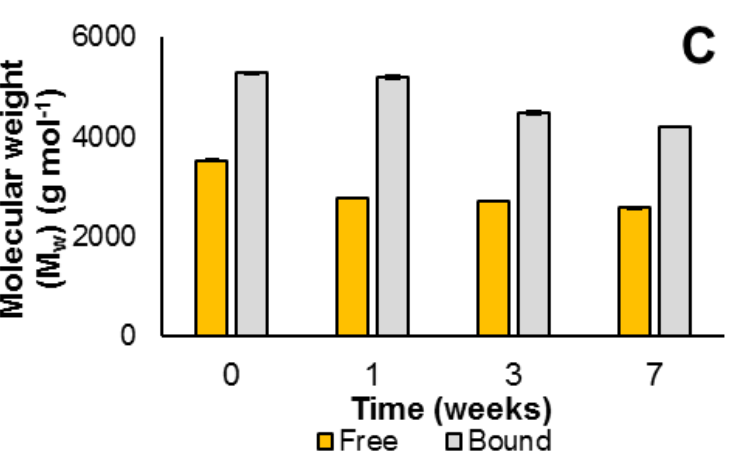

\begin{tabular}{lllll} 
C $\quad$ Bound 0 & 8200 & 29920 & 3.6 \\
$\quad$ Bound 1 & 8940 & 30340 & 3.4 \\
Bound 3 & 9025 & 32050 & 3.5 \\
Bound 7 & 8615 & 26580 & 3.1 \\
\hline
\end{tabular}

Figure S2. Normalized alkaline SEC chromatograms of free (A) and bound (B) isolates, weight average molecular weight $\left(\mathrm{M}_{\mathrm{w}}\right)$ of lignin isolates $(\mathrm{C})$. Statistical moments of molar masses of lignin isolates by organic SEC analyses calculated based on PSS standards (D) and statistical moments of molar masses of lignin isolates by organic SEC analyses calculated with MALS-corrected standards (E). Average and standard deviation in (C) of analytical duplicates of mixed isolation duplicates. For estimation of $\mathrm{M}_{\mathrm{w}}$ the small shoulders appearing in the alkaline SEC traces were excluded. 
Table S1. ${ }^{13}$ C-IS py-GC-MS relative abundance of lignin compounds in unfractionated wheat straw during growth of $C$. subvermispora. Corrected for relative response factors and relative abundance of ${ }^{13} \mathrm{C}$ analogues. Sum on the bases of structural classification according to van Erven et al. ${ }^{3-4}$ Average and standard deviation of analytical duplicates on pooled biological triplicates. Additional data (timepoints 1 and 3 weeks) compared to van Erven et al. (2018). ${ }^{3}$

\begin{tabular}{|c|c|c|c|c|}
\hline & \multicolumn{4}{|c|}{ Total sample (weeks) } \\
\hline & 0 & 1 & 3 & 7 \\
\hline \multicolumn{5}{|l|}{ Lignin subunits (\%) } \\
\hline $\mathbf{H}$ & $10.3 \pm 0.0$ & $11.7 \pm 0.3$ & $13.1 \pm 1.2$ & $15.6 \pm 0.2$ \\
\hline $\mathbf{G}$ & $64.0 \pm 0.7$ & $61.1 \pm 1.2$ & $59.6 \pm 1.0$ & $60.3 \pm 1.3$ \\
\hline $\mathbf{s}$ & $25.7 \pm 0.7$ & $27.2 \pm 0.9$ & $27.3 \pm 0.3$ & $24.1 \pm 1.1$ \\
\hline $\mathbf{S} / \mathbf{G}$ & $0.40 \pm 0.0$ & $0.44 \pm 0.0$ & $0.46 \pm 0.0$ & $0.40 \pm 0.0$ \\
\hline \multicolumn{5}{|c|}{ Structural moieties (\%) } \\
\hline Unsubstituted & $4.2 \pm 0.1$ & $4.9 \pm 0.1$ & $8.5 \pm 0.5$ & $10.7 \pm 0.3$ \\
\hline Methyl & $2.4 \pm 0.0$ & $2.2 \pm 0.1$ & $2.8 \pm 0.3$ & $3.1 \pm 0.2$ \\
\hline Vinyl & $30.9 \pm 0.5$ & $31.9 \pm 0.9$ & $34.0 \pm 0.4$ & $32.7 \pm 1.3$ \\
\hline 4-VPa & $8.7 \pm 0.0$ & $10.0 \pm 0.3$ & $10.2 \pm 1.1$ & $11.5 \pm 0.0$ \\
\hline $4-V G^{b}$ & $19.7 \pm 0.5$ & $19.3 \pm 1.3$ & $20.8 \pm 0.5$ & $18.9 \pm 1.3$ \\
\hline$C_{\alpha}-0 x$ & $3.7 \pm 0.0$ & $3.8 \pm 0.1$ & $8.2 \pm 0.2$ & $11.4 \pm 0.8$ \\
\hline diketones & $0.1 \pm 0.0$ & $0.2 \pm 0.1$ & $1.6 \pm 0.1$ & $3.1 \pm 0.6$ \\
\hline $\mathrm{C}_{\beta}-\mathrm{ox}^{\mathrm{c}}$ & $1.5 \pm 0.0$ & $1.5 \pm 0.0$ & $2.5 \pm 0.1$ & $2.8 \pm 0.2$ \\
\hline$C_{y}-0 x$ & $53.7 \pm 0.6$ & $52.5 \pm 1.2$ & $40.3 \pm 0.3$ & $35.8 \pm 0.3$ \\
\hline Miscellaneous & $3.6 \pm 0.1$ & $3.3 \pm 0.2$ & $3.7 \pm 0.0$ & $3.4 \pm 0.1$ \\
\hline $\mathrm{PhC}_{\mathrm{v}}{ }^{\mathrm{d}}$ & $58.9 \pm 0.6$ & $57.4 \pm 1.1$ & $48.8 \pm 0.2$ & $46.1 \pm 0.6$ \\
\hline $\mathrm{PhC}_{\mathrm{y}}$-diketones ${ }^{\mathrm{e}}$ & $58.8 \pm 0.6$ & $57.3 \pm 1.0$ & $47.1 \pm 0.2$ & $43.0 \pm 0.1$ \\
\hline
\end{tabular}

a 4-vinylphenol. ${ }^{\mathrm{b}} 4$-vinylguaiacol. ${ }^{\mathrm{c}}$ excluding diketones. ${ }^{\mathrm{d}}$ phenols with intact $\alpha, \beta, \gamma$ carbon side chain. ${ }^{\mathrm{e}}$ phenols with intact $\alpha, \beta, \gamma$ carbon side chain, excluding diketones. 
Table S2. Composition of free and bound lignin isolates. Lignin (\% w/w) was determined by ${ }^{13} \mathrm{C}-\mathrm{IS}$ py-GC-MS in duplicate on fractionation duplicates. Carbohydrates $(\% \mathrm{w} / \mathrm{w})$ were determined by constituent monosaccharide analysis after $\mathrm{H}_{2} \mathrm{SO}_{4}$ hydrolysis in duplicate on pooled fractionation duplicates and are presented as anhydrosugars. Values given represent averages and standard deviation. Note that ${ }^{13} \mathrm{C}-\mathrm{IS}$ py-GC-MS resulted in slight overestimation of lignin contents of bound lignin isolates (week 0 and 1).

\begin{tabular}{ccccccc}
\hline & Lignin & Glucose & Xylose & \multicolumn{2}{c}{ Arabinose } & Uronic acid Galactose \\
\hline Free lignin (weeks) & & & & & & \\
$\mathbf{0}$ & $75.1 \pm 2.5$ & $4.5 \pm 0.2$ & $18.1 \pm 1.4$ & $1.5 \pm 0.1$ & $2.1 \pm 0.1$ & $0.2 \pm 0.0$ \\
$\mathbf{1}$ & $74.7 \pm 5.8$ & $3.4 \pm 0.2$ & $18.6 \pm 1.2$ & $1.6 \pm 0.1$ & $2.0 \pm 0.1$ & $0.2 \pm 0.0$ \\
$\mathbf{3}$ & $54.8 \pm 1.6$ & $2.6 \pm 0.1$ & $15.8 \pm 1.6$ & $1.1 \pm 0.1$ & $2.0 \pm 0.1$ & $0.2 \pm 0.0$ \\
$\mathbf{7}$ & $45.0 \pm 1.3$ & $2.8 \pm 0.2$ & $12.7 \pm 0.8$ & $1.1 \pm 0.1$ & $1.7 \pm 0.1$ & $0.2 \pm 0.0$ \\
Bound lignin (weeks) & & & & & & \\
$\mathbf{0}$ & $103.7 \pm 5.4$ & $1.3 \pm 0.1$ & $10.6 \pm 0.6$ & $2.0 \pm 0.1$ & $1.4 \pm 0.2$ & $0.2 \pm 0.0$ \\
$\mathbf{1}$ & $102.1 \pm 2.2$ & $1.6 \pm 0.1$ & $10.9 \pm 0.8$ & $2.1 \pm 0.1$ & $1.3 \pm 0.2$ & $0.1 \pm 0.0$ \\
$\mathbf{3}$ & $79.7 \pm 3.9$ & $1.5 \pm 0.1$ & $11.0 \pm 0.7$ & $2.0 \pm 0.1$ & $1.2 \pm 0.2$ & $0.1 \pm 0.0$ \\
$\mathbf{7}$ & $70.8 \pm 1.8$ & $1.5 \pm 0.1$ & $10.1 \pm 0.8$ & $1.8 \pm 0.1$ & $1.1 \pm 0.2$ & $0.0 \pm 0.0$ \\
\hline
\end{tabular}


Table S3. ${ }^{13} \mathrm{C}$-IS py-GC-MS relative abundance of lignin compounds in ball-milled water extracted residue R4 of wheat straw during growth of $C$. subvermispora. Corrected for relative response factors and relative abundance of ${ }^{13} \mathrm{C}$ analogues. Sum on the bases of structural classification according to van Erven et al. ${ }^{3-4}$ Average and standard deviation of analytical duplicates on pooled biological triplicates.

\begin{tabular}{|c|c|c|c|c|}
\hline & \multicolumn{4}{|c|}{ R4 (weeks) } \\
\hline & 0 & 1 & 3 & 7 \\
\hline \multicolumn{5}{|l|}{ Lignin subunits (\%) } \\
\hline H & $11.3 \pm 1.0$ & $11.2 \pm 0.4$ & $11.7 \pm 0.6$ & $13.0 \pm 0.7$ \\
\hline $\mathbf{G}$ & $59.4 \pm 0.5$ & $58.8 \pm 0.4$ & $60.6 \pm 0.6$ & $64.3 \pm 0.4$ \\
\hline $\mathbf{S}$ & $29.3 \pm 0.9$ & $30.1 \pm 0.1$ & $27.7 \pm 0.6$ & $22.7 \pm 0.4$ \\
\hline $\mathbf{S} / \mathbf{G}$ & $0.49 \pm 0.0$ & $0.51 \pm 0.0$ & $0.46 \pm 0.0$ & $0.35 \pm 0.0$ \\
\hline \multicolumn{5}{|l|}{ Structural moieties (\%) } \\
\hline Unsubstituted & $3.9 \pm 0.3$ & $4.4 \pm 0.2$ & $6.9 \pm 0.6$ & $7.3 \pm 0.4$ \\
\hline Methyl & $2.1 \pm 0.0$ & $2.2 \pm 0.2$ & $3.4 \pm 0.8$ & $3.6 \pm 0.7$ \\
\hline Vinyl & $29.1 \pm 2.2$ & $28.6 \pm 0.9$ & $32.5 \pm 3.0$ & $34.5 \pm 0.7$ \\
\hline 4-VPa & $10.1 \pm 0.9$ & $9.9 \pm 0.4$ & $9.1 \pm 0.4$ & $9.4 \pm 0.7$ \\
\hline $4-V G^{b}$ & $17.0 \pm 1.2$ & $16.6 \pm 0.7$ & $20.3 \pm 2.5$ & $21.9 \pm 0.8$ \\
\hline $\mathrm{C}_{\alpha}-\mathrm{ox}$ & $3.9 \pm 0.2$ & $4.1 \pm 0.1$ & $7.0 \pm 0.3$ & $8.2 \pm 0.4$ \\
\hline diketones & $0.1 \pm 0.0$ & $0.2 \pm 0.0$ & $1.1 \pm 0.1$ & $1.6 \pm 0.1$ \\
\hline $\mathrm{C}_{\beta}-\mathrm{ox}^{\mathrm{c}}$ & $1.5 \pm 0.1$ & $1.5 \pm 0.1$ & $2.4 \pm 0.2$ & $2.6 \pm 0.2$ \\
\hline$C_{y}-0 x$ & $56.3 \pm 2.9$ & $55.6 \pm 1.5$ & $43.6 \pm 5.4$ & $39.3 \pm 1.4$ \\
\hline Miscellaneous & $3.5 \pm 0.3$ & $3.6 \pm 0.1$ & $4.2 \pm 0.6$ & $4.5 \pm 0.4$ \\
\hline $\mathrm{PhC}_{\mathrm{y}}{ }^{\mathrm{d}}$ & $61.3 \pm 2.6$ & $61.1 \pm 1.3$ & $51.8 \pm 4.7$ & $48.6 \pm 1.1$ \\
\hline $\mathrm{PhC}_{\mathrm{\gamma}}$-diketones ${ }^{\mathrm{e}}$ & $61.2 \pm 2.7$ & $60.9 \pm 1.3$ & $50.6 \pm 4.7$ & $47.0 \pm 1.2$ \\
\hline
\end{tabular}

a 4-vinylphenol. ${ }^{\mathrm{b}} 4$-vinylguaiacol. ${ }^{\mathrm{c}}$ excluding diketones. ${ }^{\mathrm{d}}$ phenols with intact $\alpha, \beta, \gamma$ carbon side chain. ${ }^{\mathrm{e}}$ phenols with intact $\alpha, \beta, \gamma$ carbon side chain, excluding diketones. 
Table S4. ${ }^{13} \mathrm{C}-\mathrm{IS}$ py-GC-MS relative abundance of lignin compounds in lignin isolates of wheat straw during growth of $C$. subvermispora. Corrected for relative response factors and relative abundance of ${ }^{13} \mathrm{C}$ analogues. Sum on the bases of structural classification according to van Erven et al. ${ }^{3-4}$ Average and standard deviation of analytical duplicates on pooled biological triplicates.

\begin{tabular}{|c|c|c|c|c|c|c|c|c|}
\hline & \multicolumn{4}{|c|}{ Free (weeks) } & \multicolumn{4}{|c|}{ Bound (weeks) } \\
\hline & 0 & 1 & 3 & 7 & 0 & 1 & 3 & 7 \\
\hline \multicolumn{9}{|l|}{ Lignin subunits (\%) } \\
\hline $\mathbf{H}$ & $8.6 \pm 0.1$ & $8.4 \pm 0.1$ & $8.6 \pm 0.2$ & $9.2 \pm 0.1$ & $7.9 \pm 0.1$ & $8.0 \pm 0.0$ & $7.8 \pm 0.2$ & $8.5 \pm 0.1$ \\
\hline G & $62.8 \pm 0.4$ & $62.0 \pm 0.4$ & $64.7 \pm 0.7$ & $67.2 \pm 0.8$ & $57.1 \pm 0.1$ & $57.3 \pm 0.4$ & $60.6 \pm 0.2$ & $63.7 \pm 0.3$ \\
\hline S & $28.6 \pm 0.4$ & $29.6 \pm 0.4$ & $26.6 \pm 0.7$ & $23.6 \pm 0.6$ & $35.0 \pm 0.1$ & $34.7 \pm 0.4$ & $31.7 \pm 0.1$ & $27.8 \pm 0.5$ \\
\hline $\mathbf{S} / \mathbf{G}$ & $0.45 \pm 0.0$ & $0.48 \pm 0.0$ & $0.41 \pm 0.0$ & $0.35 \pm 0.0$ & $0.61 \pm 0.0$ & $0.61 \pm 0.0$ & $0.52 \pm 0.0$ & $0.44 \pm 0.0$ \\
\hline \multicolumn{9}{|c|}{ Structural moieties (\%) } \\
\hline Unsubstituted & $4.6 \pm 0.1$ & $4.5 \pm 0.0$ & $5.8 \pm 0.0$ & $6.8 \pm 0.2$ & $3.7 \pm 0.1$ & $3.8 \pm 0.0$ & $4.8 \pm 0.1$ & $5.0 \pm 0.4$ \\
\hline Methyl & $1.5 \pm 0.0$ & $1.6 \pm 0.0$ & $1.9 \pm 0.0$ & $2.0 \pm 0.1$ & $1.5 \pm 0.0$ & $1.6 \pm 0.0$ & $1.9 \pm 0.1$ & $2.0 \pm 0.1$ \\
\hline Vinyl & $18.8 \pm 0.2$ & $19.0 \pm 0.0$ & $19.0 \pm 0.3$ & $19.1 \pm 0.5$ & $19.8 \pm 0.2$ & $20.1 \pm 0.1$ & $21.5 \pm 0.2$ & $22.5 \pm 0.5$ \\
\hline 4-VPa & $6.8 \pm 0.0$ & $6.5 \pm 0.2$ & $6.2 \pm 0.2$ & $6.4 \pm 0.4$ & $6.8 \pm 0.1$ & $6.9 \pm 0.1$ & $6.3 \pm 0.1$ & $6.9 \pm 0.2$ \\
\hline $4-V^{b}$ & $10.3 \pm 0.2$ & $10.5 \pm 0.2$ & $11.0 \pm 0.1$ & $11.3 \pm 0.1$ & $10.7 \pm 0.1$ & $11.0 \pm 0.1$ & $12.9 \pm 0.3$ & $13.5 \pm 0.3$ \\
\hline $\mathrm{C}_{\alpha}-\mathrm{ox}$ & $3.9 \pm 0.1$ & $4.2 \pm 0.1$ & $6.7 \pm 0.1$ & $9.0 \pm 0.1$ & $3.4 \pm 0.0$ & $3.6 \pm 0.0$ & $5.1 \pm 0.1$ & $5.3 \pm 0.0$ \\
\hline diketones & $0.1 \pm 0.0$ & $0.2 \pm 0.0$ & $0.7 \pm 0.0$ & $1.8 \pm 0.1$ & $0.1 \pm 0.0$ & $0.1 \pm 0.0$ & $0.5 \pm 0.0$ & $0.7 \pm 0.0$ \\
\hline $\mathrm{C}_{\beta}-\mathrm{ox}^{\mathrm{c}}$ & $1.3 \pm 0.0$ & $1.3 \pm 0.0$ & $1.7 \pm 0.0$ & $1.8 \pm 0.1$ & $1.2 \pm 0.0$ & $1.2 \pm 0.0$ & $1.6 \pm 0.0$ & $1.6 \pm 0.0$ \\
\hline$C_{y}-0 x$ & $67.0 \pm 0.3$ & $66.4 \pm 0.1$ & $61.9 \pm 0.4$ & $59.3 \pm 0.2$ & $67.4 \pm 0.3$ & $66.6 \pm 0.1$ & $62.0 \pm 0.3$ & $60.6 \pm 0.8$ \\
\hline Miscellaneous & $2.8 \pm 0.0$ & $2.9 \pm 0.0$ & $3.0 \pm 0.0$ & $3.1 \pm 0.0$ & $2.9 \pm 0.0$ & $3.0 \pm 0.0$ & $3.1 \pm 0.0$ & $3.1 \pm 0.0$ \\
\hline $\mathrm{PhCY}^{\mathrm{d}}$ & $71.8 \pm 0.3$ & $71.4 \pm 0.1$ & $68.4 \pm 0.4$ & $66.4 \pm 0.2$ & $72.0 \pm 0.3$ & $71.3 \pm 0.1$ & $67.7 \pm 0.3$ & $66.5 \pm 0.7$ \\
\hline $\mathrm{PhC}_{\mathrm{r}}$-diketones ${ }^{\mathrm{e}}$ & $71.7 \pm 0.3$ & $71.2 \pm 0.1$ & $67.7 \pm 0.4$ & $64.5 \pm 0.2$ & $71.9 \pm 0.3$ & $71.2 \pm 0.1$ & $67.2 \pm 0.3$ & $66.0 \pm 0.7$ \\
\hline
\end{tabular}

${ }^{\mathrm{a}}$ 4-vinylphenol. ${ }^{\mathrm{b}}$ 4-vinylguaiacol. ${ }^{\mathrm{c}}$ excluding diketones. ${ }^{\mathrm{d}}$ phenols with intact $\alpha, \beta, \gamma$ carbon side chain. ${ }^{\mathrm{e}}$ phenols with intact $\alpha, \beta, \gamma$ carbon side chain, excluding diketones. 
Table S5. ${ }^{13} \mathrm{C}$-IS py-GC-MS relative abundance of lignin compounds in unextractable residue R7 of wheat straw during growth of $C$. subvermispora. Corrected for relative response factors and relative abundance of ${ }^{13} \mathrm{C}$ analogues. Sum on the bases of structural classification according to van Erven et al. ${ }^{3-4}$ Average and standard deviation of analytical duplicates on pooled biological triplicates.

\begin{tabular}{|c|c|c|c|c|}
\hline & \multicolumn{4}{|c|}{ R7: dioxane insoluble residue (weeks) } \\
\hline & 0 & 1 & 3 & 7 \\
\hline \multicolumn{5}{|l|}{ Lignin subunits (\%) } \\
\hline $\mathbf{H}$ & $12.7 \pm 0.3$ & $13.3 \pm 0.6$ & $12.4 \pm 0.4$ & $14.8 \pm 0.6$ \\
\hline G & $55.3 \pm 0.5$ & $54.7 \pm 0.3$ & $56.8 \pm 0.3$ & $60.2 \pm 1.0$ \\
\hline S & $32.1 \pm 0.6$ & $32.0 \pm 1.0$ & $30.7 \pm 0.7$ & $24.9 \pm 0.5$ \\
\hline $\mathbf{S} / \mathbf{G}$ & $0.58 \pm 0.0$ & $0.58 \pm 0.0$ & $0.54 \pm 0.0$ & $0.41 \pm 0.0$ \\
\hline \multicolumn{5}{|c|}{ Structural moieties (\%) } \\
\hline Unsubstituted & $4.5 \pm 0.1$ & $4.9 \pm 0.1$ & $6.3 \pm 0.3$ & $7.4 \pm 0.5$ \\
\hline Methyl & $2.5 \pm 0.1$ & $2.5 \pm 0.1$ & $2.8 \pm 0.2$ & $3.5 \pm 0.1$ \\
\hline Vinyl & $27.1 \pm 0.4$ & $27.7 \pm 1.1$ & $27.9 \pm 0.7$ & $31.2 \pm 1.3$ \\
\hline 4-VPa & $10.8 \pm 0.3$ & $11.3 \pm 0.5$ & $9.9 \pm 0.3$ & $11.2 \pm 0.7$ \\
\hline $4-V G^{b}$ & $13.5 \pm 0.1$ & $13.7 \pm 0.5$ & $15.0 \pm 0.4$ & $17.2 \pm 0.5$ \\
\hline $\mathrm{C}_{\alpha}-\mathrm{ox}$ & $3.6 \pm 0.1$ & $3.9 \pm 0.2$ & $6.0 \pm 0.1$ & $7.0 \pm 0.2$ \\
\hline diketones & $0.1 \pm 0.0$ & $0.2 \pm 0.0$ & $0.9 \pm 0.1$ & $1.2 \pm 0.1$ \\
\hline $\mathrm{C}_{\beta}-\mathrm{ox}^{\mathrm{c}}$ & $1.7 \pm 0.1$ & $1.7 \pm 0.1$ & $2.2 \pm 0.1$ & $2.6 \pm 0.1$ \\
\hline$C_{y}-0 x$ & $56.7 \pm 0.5$ & $55.3 \pm 1.6$ & $50.8 \pm 1.2$ & $44.1 \pm 1.5$ \\
\hline Miscellaneous & $4.0 \pm 0.0$ & $4.0 \pm 0.3$ & $3.9 \pm 0.1$ & $4.2 \pm 0.1$ \\
\hline $\mathrm{PhC}_{\mathrm{y}}{ }^{\mathrm{d}}$ & $62.5 \pm 0.4$ & $61.3 \pm 1.3$ & $58.3 \pm 1.0$ & $52.6 \pm 1.5$ \\
\hline $\mathrm{PhC}_{\mathrm{y}}$-diketones & $62.4 \pm 0.4$ & $61.1 \pm 1.3$ & $57.4 \pm 1.0$ & $51.4 \pm 1.4$ \\
\hline
\end{tabular}

a 4-vinylphenol. ${ }^{\mathrm{b}} 4$-vinylguaiacol. ${ }^{\mathrm{c}}$ excluding diketones. ${ }^{\mathrm{d}}$ phenols with intact $\alpha, \beta, \gamma$ carbon side chain. ${ }^{\mathrm{e}}$ phenols with intact $\alpha, \beta, \gamma$ carbon side chain, excluding diketones. 
Table S6. Assignments of the lignin ${ }^{13} \mathrm{C}-{ }^{1} \mathrm{H}$ correlation peaks in the HSQC spectra of untreated and fungaltreated wheat straw lignin fractions.

\begin{tabular}{|c|c|c|}
\hline label & $\delta_{\mathrm{C}} / \delta_{\mathrm{H}}(\mathrm{ppm})$ & Assignment $^{\mathrm{a}}$ \\
\hline CHK & $51.5 / 3.58$ & $\mathrm{C}-\mathrm{H}$ in methoxyls of cyclohexadienone ketals $(\mathrm{t})$ \\
\hline$\underline{\mathrm{B}_{\beta}}$ & $53.0 / 3.43$ & $\mathrm{C}_{\beta}-\mathrm{H}_{\beta}$ in phenylcoumaran substructures \\
\hline$\underline{\mathrm{C}_{\beta}}$ & $53.6 / 3.05$ & $\mathrm{C}_{\beta}-\mathrm{H}_{\beta}$ in resinol substructures \\
\hline$-\mathrm{OCH}_{3}$ & $55.6 / 3.72$ & $\mathrm{C}-\mathrm{H}$ in methoxyls \\
\hline $\mathrm{A}_{\gamma}$ & $59.6 / 3.37,59.8 / 3.59,59.9 / 3.22$ & $\mathrm{C}_{Y}-\mathrm{H}_{Y}$ in $\beta-O-4^{\prime}$ substructures \\
\hline$\underline{I_{y}}$ & $61.4 / 4.09$ & $\mathrm{C}_{\gamma}-\mathrm{H}_{\gamma}$ in cinnamyl alcohol end-groups \\
\hline$\underline{A_{Y}^{\prime}}$ & $62.8 / 4.23,63.9 / 4.18$ & $\mathrm{C}_{\gamma}-\mathrm{H}_{\gamma}$ in $\gamma$-acylated $\beta$-O-4' substructures \\
\hline $\mathrm{C}_{y}$ & $71.0 / 4.16,71.1 / 3.80$ & $\mathrm{C}_{\beta}-\mathrm{H}_{\beta}$ in resinol substructures \\
\hline$\underline{\mathrm{A}_{\alpha}(\mathrm{G})}$ & $70.9 / 4.71$ & $\mathrm{C}_{\alpha}-\mathrm{H}_{\alpha}$ in $\beta-O-4$ ' substructures linked to G-unit \\
\hline$\underline{\mathrm{A}_{\alpha}(\mathrm{S})}$ & $71.8 / 4.81$ & $\mathrm{C}_{\alpha}-\mathrm{H}_{\alpha}$ in $\beta-O-4$ ' substructures linked to S-unit \\
\hline$\underline{A_{o \times \beta}}$ & $82.8 / 5.10$ & $\mathrm{C}_{\beta}-\mathrm{H}_{\beta}$ in $\mathrm{C}_{\alpha}$-oxidized $\beta$-O-4' substructures \\
\hline$\underline{\mathrm{A}_{\beta}(\mathrm{H})}$ & $83.1 / 4.49$ & $\mathrm{C}_{\beta}-\mathrm{H}_{\beta}$ in $\beta-O-4$ ' substructures linked to $\mathrm{H}$-unit \\
\hline$\underline{A_{\beta}(G)}$ & $83.5 / 4.27$ & $\mathrm{C}_{\beta}-\mathrm{H}_{\beta}$ in $\beta-O-4$ ' substructures linked to G-unit \\
\hline$\underline{A_{\beta}(S)_{\text {erythro }}}$ & $85.9 / 4.09$ & $\mathrm{C}_{\beta}-\mathrm{H}_{\beta}$ in $\beta-O-4^{\prime}$ substructures linked to S-unit \\
\hline$\underline{A_{\beta}(T)}$ & $86.2 / 4.36$ and $86.7 / 4.26$ & $\mathrm{C}_{\beta}-\mathrm{H}_{\beta}$ in $\beta-O-4^{\prime}$ substructures linked to tricin \\
\hline$\underline{A_{\beta}(S)_{\text {threo }}}$ & $86.9 / 3.97$ & $\mathrm{C}_{\beta}-\mathrm{H}_{\beta}$ in $\beta-O-4^{\prime}$ substructures linked to S-unit \\
\hline$\underline{\mathrm{C}_{\alpha}}$ & $84.9 / 4.64$ & $\mathrm{C}_{\alpha}-\mathrm{H}_{\alpha}$ in resinol substructures \\
\hline$\underline{\mathrm{B}_{\alpha}}$ & $86.9 / 5.43$ & $\mathrm{C}_{\alpha}-\mathrm{H}_{\alpha}$ in phenylcoumaran substructures \\
\hline$\underline{T_{8}}$ & $94.1 / 6.57$ & $\mathrm{C}_{8}-\mathrm{H}_{8}$ in tricin \\
\hline$\underline{\mathrm{T}_{6}}$ & $98.8 / 6.21$ & $\mathrm{C}_{6}-\mathrm{H}_{6}$ in tricin \\
\hline$\underline{T_{\text {free3 }}}$ & $103.5 / 6.96$ & $\mathrm{C}_{3}-\mathrm{H}_{3}$ in free tricin \\
\hline$\underline{\mathrm{S}_{2,6}}$ & $103.9 / 6.68$ & $\mathrm{C}_{2}-\mathrm{H}_{2}$ and $\mathrm{C}_{6}-\mathrm{H}_{6}$ in S-unit \\
\hline$\underline{T_{2^{\prime} 6^{\prime}} / T_{\text {free2' } 6^{\prime}}}$ & $104.0 / 7.31$ & $\mathrm{C}_{2^{\prime}-}-\mathrm{H}_{2^{\prime}}$ and $\mathrm{C}_{6^{\prime}} \cdot \mathrm{H}_{6^{\prime}}$ in tricin and in free tricin \\
\hline$\underline{T_{3}}$ & $104.6 / 7.02$ & $\mathrm{C}_{3}-\mathrm{H}_{3}$ in tricin \\
\hline Sox $_{2,6}$ & $106.4 / 7.30$ & $\mathrm{C}_{2}-\mathrm{H}_{2}$ and $\mathrm{C}_{6}-\mathrm{H}_{6}$ in $\mathrm{C}_{\mathrm{a}}$-oxidized $\left(\mathrm{C}_{\mathrm{a}}=\mathrm{O}\right)$ S-unit \\
\hline$\underline{\text { Sox }_{2,6}}$ & $106.5 / 7.19$ & $\mathrm{C}_{2}-\mathrm{H}_{2}$ and $\mathrm{C}_{6}-\mathrm{H}_{6}$ in $\mathrm{C}_{\mathrm{a}}$-oxidized $\left(\mathrm{C}_{\mathrm{a}} \mathrm{OOH}\right)$ S-unit \\
\hline$\underline{\mathrm{G}_{2}}$ & $110.8 / 6.96$ & $\mathrm{C}_{2}-\mathrm{H}_{2}$ in G-unit \\
\hline $\mathrm{FA}_{2}$ & $110.9 / 7.34$ & $\mathrm{C}_{2}-\mathrm{H}_{2}$ in ferulate \\
\hline $\mathrm{Goxl}_{2}$ & $111.4 / 7.51$ & $\mathrm{C}_{2}-\mathrm{H}_{2}$ in $\mathrm{C}_{\mathrm{a}}$-oxidized G-unit \\
\hline $\mathrm{Goxll}_{2}$ & $112.4 / 7.44$ & $\mathrm{C}_{2}-\mathrm{H}_{2}$ in $\mathrm{C}_{\mathrm{a}}$-oxidized G-unit \\
\hline$\underline{F A_{\beta} / p C A_{\beta}}$ & $113.6 / 6.26$ & $\mathrm{C}_{\beta}-\mathrm{H}_{\beta}$ in ferulate $/ p$-coumarate \\
\hline$\underline{\mathrm{H}_{3,5} / \mathrm{FA}_{5}}$ & $114.5 / 6.69$ & $\mathrm{C}_{3}-\mathrm{H}_{3}$ and $\mathrm{C}_{5}-\mathrm{H}_{5}$ in H-unit, $\mathrm{C}_{5}-\mathrm{H}_{5}$ in $\mathrm{FA}$ \\
\hline$\underline{\mathrm{G}_{5} / \mathrm{G}_{6} / \mathrm{pCA}_{3,5}}$ & $115.0 / 6.93$ and $115.5 / 6.78$ & $\mathrm{C}_{5}-\mathrm{H}_{5}$ and $\mathrm{C}_{6}-\mathrm{H}_{6}$ in G-unit, $\mathrm{C}_{3}-\mathrm{H}_{3}$ and $\mathrm{C}_{5}-\mathrm{H}_{5}$ of pCA \\
\hline $\mathrm{G}_{5}$ & 119.0/6.78 & $\mathrm{C}_{5}-\mathrm{H}_{5}$ in G-unit \\
\hline $\mathrm{Goxl}_{6}$ & $122.7 / 7.47$ & $\mathrm{C}_{6}-\mathrm{H}_{6}$ in $\mathrm{C}_{\mathrm{a}}$-oxidized G-unit \\
\hline $\mathrm{FA}_{6}$ & $123.1 / 7.12$ & $\mathrm{C}_{6}-\mathrm{H}_{6}$ in ferulate \\
\hline$\underline{G o x l l}_{6}$ & $125.8 / 7.42$ & $\mathrm{C}_{2}-\mathrm{H}_{2}$ in $\mathrm{C}_{\mathrm{a}}$-oxidized G-unit \\
\hline$\underline{\mathrm{H}_{2,6} / \mathrm{PHE}_{3,5}}$ & $127.8 / 7.18$ & $\mathrm{C}_{2}-\mathrm{H}_{2}$ and $\mathrm{C}_{6}-\mathrm{H}_{6}$ in $\mathrm{H}$-unit, $\mathrm{C}_{3}-\mathrm{H}_{3}$ and $\mathrm{C}_{5}-\mathrm{H}_{5}$ in phenylalanine \\
\hline $\mathrm{PHE}_{2,6}$ & $128.9 / 7.21$ & $\mathrm{C}_{2}-\mathrm{H}_{2}$ and $\mathrm{C}_{6}-\mathrm{H}_{6}$ in phenylalanine \\
\hline $\mathrm{pCA}_{2,6}$ & $130.1 / 7.42$ & $\mathrm{C}_{2}-\mathrm{H}_{2}$ and $\mathrm{C}_{6}-\mathrm{H}_{6}$ in $p$-coumarate \\
\hline $\mathrm{FA}_{\alpha} / \mathrm{pCA}_{\alpha}$ & $145.1 / 7.57$ & $\mathrm{C}_{\alpha}-\mathrm{H}_{\alpha}$ in ferulate/p-coumarate \\
\hline
\end{tabular}

a: assignment by comparison with literature. ${ }^{4-11}(\mathrm{t})$ : tentatively assigned 
Table S7. Semi-quantitative HSQC NMR structural characterization of wheat straw lignin isolates in the absence and presence of chromium (III) acetylacetonate $\left(\mathrm{Cr}(\mathrm{acac})_{3}\right)$ as relaxation agent. Note that the untreated and $C$. subvermispora treated wheat straw lignin isolates shown here originate from a different batch of straw and should therefore solely be used for comparisons of $\mathrm{Cr}(\mathrm{acac})_{3}$ effects.

\begin{tabular}{|c|c|c|c|c|}
\hline & \multicolumn{2}{|c|}{ Untreated wheat straw (free + bound) } & \multicolumn{2}{|c|}{7 weeks fungal treated wheat straw (free) } \\
\hline & Without $\operatorname{Cr}(\text { acac })_{3}$ & With $\mathrm{Cr}(\mathrm{acac})_{3}$ & Without $\operatorname{Cr}(\text { acac })_{3}$ & With $\mathrm{Cr}(\mathrm{acac})_{3}$ \\
\hline \multicolumn{5}{|l|}{ Lignin subunits $(\%)^{\mathrm{a}}$} \\
\hline $\mathbf{H}$ & 3 & 3 & 3 & 2 \\
\hline $\mathbf{G}$ & 62 & 63 & 53 & 55 \\
\hline $\mathrm{G}_{\mathrm{ox}}$ & 0 & 0 & 10 & 11 \\
\hline $\mathbf{S}$ & 35 & 34 & 24 & 23 \\
\hline$S_{o x}$ & 0 & 0 & 9 & 9 \\
\hline $\mathbf{S} / \mathbf{G}$ & 0.6 & 0.5 & 0.5 & 0.5 \\
\hline \multicolumn{5}{|l|}{ Hydroxycinnamates $(\%)^{b}$} \\
\hline p-coumarate & 8 & 6 & 17 & 13 \\
\hline ferulate & 5 & 3 & 6 & 4 \\
\hline \multicolumn{5}{|l|}{ Flavonolignin (\%) ${ }^{\mathrm{b}}$} \\
\hline tricin & 15 & 9 & 21 & 15 \\
\hline \multicolumn{5}{|l|}{ Lignin interunit linkages $(\%)^{b, c}$} \\
\hline$\beta-O-4^{\prime} G+H$ & $20(35)$ & $22(37)$ & $18(38)$ & $19(38)$ \\
\hline$\beta-0-4$ 'S & $22(38)$ & $24(40)$ & $14(29)$ & $16(32)$ \\
\hline$\beta-0-4{ }^{\prime} C_{\alpha}-0 x$ & $1(1)$ & $1(2)$ & $0(0)$ & $0(0)$ \\
\hline$\beta-O-4$ ' tricin & $8(15)$ & $8(13)$ & $11(22)$ & $11(23)$ \\
\hline total $\beta-0-4$ ' aryl ethers & $51(89)$ & $55(92)$ & $43(89)$ & $46(93)$ \\
\hline$\beta-5$ ' phenylcoumarans & $5(8)$ & $4(6)$ & $3(7)$ & $2(5)$ \\
\hline$\beta-\beta$ ' resinols & $1(2)$ & $1(2)$ & $2(4)$ & $1(2)$ \\
\hline total & $57(100)$ & $60(100)$ & $49(100)$ & $50(100)$ \\
\hline Side-chain $\mathrm{y}$-acylation (\%) ${ }^{d}$ & 21 & 20 & 17 & 16 \\
\hline Erythro/threo of $\beta-O-4^{e}$ & 2.6 & 2.5 & 3.9 & 4.0 \\
\hline
\end{tabular}

${ }^{a}$ relative distribution of lignin subunits $\left(\mathrm{H}+\mathrm{G}+\mathrm{G}_{\mathrm{ox}}+\mathrm{S}+\mathrm{S}_{\mathrm{ox}}=100\right)$

${ }^{b}$ relative volume integral of substructure versus volume integral of total lignin subunits

${ }^{c}$ relative distribution of total interunit linkages in parentheses

d percentage of $\beta-O-4$ ' aryl ethers

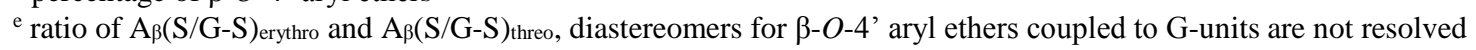


Table S8. Hydroxyl group content of lignin isolates of wheat straw during growth of C. subvermispora determined by ${ }^{31} \mathrm{P}$ NMR after phosphitylation.

\begin{tabular}{ccccc|cccc}
\hline & \multicolumn{3}{c|}{ Free (weeks) (mmol/g lignin) $^{\mathrm{a}}$} & \multicolumn{4}{c}{ Bound (weeks) (mmol/g lignin) $^{\mathrm{a}}$} \\
\hline Aliphatic OH + carb. OH & $\mathbf{0}$ & $\mathbf{1}$ & $\mathbf{3}$ & $\mathbf{7}$ & $\mathbf{0}$ & $\mathbf{1}$ & $\mathbf{3}$ & $\mathbf{7}$ \\
S-OH + 5-sub. G-OH & 0.56 & 0.62 & 0.95 & 1.23 & 0.39 & 0.43 & 0.63 & 0.71 \\
G-OH & 0.74 & 0.72 & 0.88 & 1.01 & 0.53 & 0.53 & 0.61 & 0.71 \\
H-OH + pCA-OH & 0.57 & 0.57 & 0.78 & 0.93 & 0.42 & 0.42 & 0.53 & 0.65 \\
Total phenolic OH & 1.87 & 1.91 & 2.61 & 3.17 & 1.34 & 1.38 & 1.76 & 2.07 \\
COOH & 0.28 & 0.36 & 0.79 & 1.33 & 0.14 & 0.17 & 0.34 & 0.43 \\
\hline
\end{tabular}

${ }^{\mathrm{a}}$ contents per $\mathrm{g}$ biomass corrected for lignin contents as determined by quantitative ${ }^{13} \mathrm{C}-\mathrm{IS}$ py-GC-MS 


\section{References}

1. Englyst, H. N.; Cummings, J. H., Simplified method for the measurement of total non-starch polysaccharides by gas-liquid chromatography of constituent sugars as alditol acetates. Analyst 1984, 109 (7), 937-942.

2. Blumenkrantz, N.; Asboe-Hansen, G., New method for quantitative determination of uronic acids. Analytical biochemistry 1973, 54 (2), 484-489.

3. van Erven, G.; Nayan, N.; Sonnenberg, A. S.; Hendriks, W. H.; Cone, J. W.; Kabel, M. A., Mechanistic insight in the selective delignification of wheat straw by three white-rot fungal species through quantitative ${ }^{13} \mathrm{C}$ IS py-GC-MS and whole cell wall HSQC NMR. Biotechnol Biofuels 2018, 11, 262.

4. van Erven, G.; Hilgers, R.; de Waard, P.; Gladbeek, E.-J.; van Berkel, W. J.; Kabel, M. A., Elucidation of in situ ligninolysis mechanisms of the selective white-rot fungus Ceriporiopsis subvermispora. ACS Sustainable Chem Eng 2019, forthcoming, DOI: 10.1021/acssuschemeng.9b04235.

5. Ralph, S. A.; Ralph, J.; Landucci, L., NMR database of lignin and cell wall model compounds. 2009, Available at URL www.glbrc.org/databases and software/nmrdatabasel.

6. Del Río, J. C.; Rencoret, J.; Prinsen, P.; Martínez, A. T.; Ralph, J.; Gutiérrez, A., Structural characterization of wheat straw lignin as revealed by analytical pyrolysis, 2D-NMR, and reductive cleavage methods. J Agric Food Chem 2012, 60, 5922-5935.

7. Rencoret, J.; Gutiérrez, A.; Nieto, L.; Jiménez-Barbero, J.; Faulds, C. B.; Kim, H.; Ralph, J.; Martínez, Á. T.; José, C., Lignin composition and structure in young versus adult Eucalyptus globulus plants. Plant Physiol 2011, 155, 667-682.

8. $\quad$ Rencoret, J.; Pereira, A.; del Río, J. C.; Martínez, Á. T.; Gutiérrez, A., Delignification and saccharification enhancement of sugarcane byproducts by a laccase-based pretreatment. ACS Sustainable Chem Eng 2017, 5, 7145-7154.

9. Del Río, J. C.; Lino, A. G.; Colodette, J. L.; Lima, C. F.; Gutiérrez, A.; Martínez, Á. T.; Lu, F.; Ralph, J.; Rencoret, J., Differences in the chemical structure of the lignins from sugarcane bagasse and straw. Biomass Bioenergy 2015, 81, 322-338.

10. Kim, H.; Padmakshan, D.; Li, Y.; Rencoret, J.; Hatfield, R. D.; Ralph, J., Characterization and elimination of undesirable protein residues in plant cell wall materials for enhancing lignin analysis by solutionstate nuclear magnetic resonance spectroscopy. Biomacromolecules 2017, 18, 4184-4195.

11. Lan, W.; Yue, F.; Rencoret, J.; del Río, J.; Boerjan, W.; Lu, F.; Ralph, J., Elucidating tricin-lignin structures: assigning correlations in HSQC spectra of monocot lignins. Polymers 2018, 10, 916. 